\title{
Baroque
}

BAROQUE

\section{Écriture expressionniste et théâtralité dans le récit picaresque}

Edmond Cros

\section{(2) OpenEdition \\ 12 Journals}

Édition électronique

URL : http://journals.openedition.org/baroque/515

DOI : $10.4000 /$ baroque. 515

ISSN : 2261-639X

Éditeur :

Centre de recherches historiques - EHESS, Éditions Cocagne

\section{Édition imprimée}

Date de publication : 1 mai 1980

ISSN : 0067-4222

\section{Référence électronique}

Edmond Cros, «Écriture expressionniste et théâtralité dans le récit picaresque », Baroque [En ligne], 09-10 | 1980, mis en ligne le 13 mai 2013, consulté le 03 mai 2019. URL : http://

journals.openedition.org/baroque/515; DOI : 10.4000/baroque.515

Ce document a été généré automatiquement le 3 mai 2019.

(c) Tous droits réservés 


\title{
Écriture expressionniste et théâtralité dans le récit picaresque
}

\author{
Edmond Cros
}

\section{Communication}

1 La composition et l'écriture des romans picaresques espagnols se situent au confluent de la littérature populaire et de la création savante. D'une part, en effet, c'est à l'autorité plus ou moins étroite de la rhétorique traditionnelle que se soumettent le Lazarillo, le Guzmán et le Buscón. D’autre part, les épisodes narratifs tout autant que la typologie des personnages relèvent souvent de traditions folkloriques. Les différents thèmes populaires redistribués par le Lazarillo ont été étudiés par Marcel Bataillon, dont l'étude a été récemment complétée par celle de Lazaro Carreter. Or toute cette matière narrative traditionnelle se coule dans le cadre d'une formule autobiographique qui s'inspire d'un type de lettre fréquente pendant la Renaissance, du moins en Espagne et que l'on appelle les lettres-dialogues, où, à la demande d'un ami, l'auteur raconte et décrit une situation personnelle ou faussement personnelle, lettres toujours introduites par la même formule, «Vuestra merced escrive, se le escribe.... ( ( Vous m'écrivez que je dois vous écrire... »), qui n'est elle même que la traduction d'un stéréotype latin. Mateo Aleman puise, à pleines mains, dans les proverbes populaires et les traditions folkloriques, qui sont véhiculées par les fabliaux, les contes, les nouvelles italiennes, les recueils de facéties de tous ordres ou encore par l'expression orale mais chez lui la médiation de la rhétorique est essentielle; c'est cette médiation qui régit la conduite de la démonstration picaresque qui s'organise autour de deux lieux intrinsèques de la Rhétorique, qui sont le lieu du contraire et le lieu de la cause et de l'effet et c'est à cette médiation que la description, chez Mateo Aleman, doit de se confondre avec ce qu'on pourrait appeler l'art « d'égarer les cœurs et les esprits ». Or cette médiation n'est qu'un reflet de ce qu'on peut considérer comme étant l'axe conceptuel de l'ouvrage, c'est-à-dire la dialectique de la Justice et de la Miséricorde. Dans une sorte de combinaison inversée par rapport au Guzmán, c'est de l'utilisation d'un système emprunté à la littérature populaire, carnavalesque que doit être rapproché ce qui constitue l'ace conceptuel du Buscón c'est-à-dire une opposition entre la mystification et 
la démystification tandis que, toujours dans le Buscón, c'est une fonction beaucoup plus subtile qui est réservée à la rhétorique, dans la mesure où celle-ci est conçue comme l'indice de l'usurpation d'un certain type de discours.

Les modalités de cette combinaison entre la littérature populaire et la création savante devraient être analysées dans le cadre de la distinction aristotélicienne des trois genres, car cette canalisation d'une matière "humble» dans le moule des stéréotypes d'un discours «noble » est un facteur d'éventuelle distorsion dans le domaine de la création artistique et on peut se demander s'il ne s'agit pas d'une fissure par où a pu s'introduire et s'insinuer une certaine forme de modernité. Lorsqu'on analyse le Guzmán et le Buscón dans ce cadre général et en essayant de réfléchir sur ce qu'est la pensée baroque, on observe que l'écriture du Guzmán relève d'une technique expressioniste qui procède directement de la rhétorique traditionnelle et ceci à travers les procédés d'amplification d'une part et l'application du système des affectus d'autre part. De tous les procédés d'amplification je retiendrai entre autres cette loi rappelée par Horace dans l'Épitre aux Pisons, qui établit les rapports d'homologie entre le dedans et le dehors. "Car la nature nous modèle d'abord au-dedans selon tous les états divers de la fortune; elle nous inspire la joie, nous pousse à la colère puis elle relève au dehors les mouvements de l'âme, en prenant la langue comme interprète ». Ce théorème procède du sentiment que l'attitude du personnage et les paroles qu'il prononce doivent être en harmonie avec son état d'âme, mais ce système relativement primaire peut s'enrichir ; ces rapports analogiques qui se dégagent d'une observation superficielle peuvent en effet se doubler en profondeur d'un couple antithétique où on opposera les apparences extérieures à la réalité interne, couple qui est très exactement le négatif de ce premier.

Dans le récit picaresque c'est ce négatif qui l'emporte, sous l'influence probable des courants exemplaires. Citons ici deux exemples du Guzmán, il s'agit pour le premier d'une anecdote qui met en scène une jeune femme simulant une maladie pour pouvoir rejoindre son amant : «Force lui fut de se laisser tomber de la selle où, sur son ânon de Sardaigne elle était assise, avec de telles démonstrations de douleur, des grimaces et des contorsions, se serrant le ventre, se tordant les mains, dodelinant de la tête, dégrafant son corsage qu'elle trompa tout le monde et qu'à tous elle faisait pitié »... Le second exemple tiré d'une histoire intercalée qui est constituée par une très belle nouvelle mauresque décrit un jeune errant au moment où il apprend que sa fiancée vient d'être capturée par les chrétiens; "Dès que son époux l'apprit, quels airs jeta-t-il, quelles plaintes exhala-t-il, quels soupirs poussa-t-il, quels effets de sa tristesse montra-t-il ?» Voilà deux bons exercices sur la douleur physique et la douleur morale dont la juxtaposition met parfaitement en relief la technique de Mateo Aleman et ses insuffisances. La douleur feinte dans le premier cas et celle qui est véritable dans le second, la souffrance physique et l'affliction morale sont perçues de façon identique, c'est-à-dire appréhendées de l'extérieur dans des visions conventionnelles et vides de tout contenu : ces analyses aboutissent à d'heureux effets dans les cas où comme dans le premier passage (les simulations de la maladie), tout n'est que feinte, simulation, hypocrisie et peut-être n'est-il pas vain de chercher dans ces témoignages à peine voilés d'habitudes scolaires, dans cette ambiance saturée d'études académiques, les raisons pour lesquelles ont pu se développer aussi rigoureusement et aussi largement dans la prose et dans la poésie du Siècle d'or les thèmes de la désillusion et de l'hypocrisie, ce refus des apparences, cette méfiance des perceptions sensibles que l'un et l'autre supposent. On peut se demander s'il n'y a pas un rapport, direct ou indirect, entre d'une part la prise de 
conscience de ce que cette équation première entre l'intérieur et l'extérieur cache souvent une antithèse et, d'autre part, la métaphore "conceptiste ", qui caractérise la poésie dite baroque. Celle-ci en effet, comme l'a fait observer Léo Sptizer effleure l'objet dont elle se limite à décrire les contours (de nos jours nous parlerions de connotations). Et si les apparences de l'objet sont fausses ou du moins douteuses, la tentation n'est-elle pas grande de se détourner de lui pour en trouver l'essence, qui se trouve au-delà de lui ? La métaphore baroque pourrait être ainsi l'indice d'une problématique culturelle basée sur un sentiment d'instabilité et d'insécurité créé par la perception d'une sorte de vide. Dans ces conditions, quel est le point d'ancrage de la description dans le Guzmán : lorsque nous parlions de connotations, nous évoquions implicitement la présence d'un destinataire ce qui nous amène à parler du système des affectus, qui constitue un des axes principaux de l'esthétique proposée par la rhétorique. Ce concept domine l'organisation de la description dans le Guzmán et il assujettit l'expression métaphorique qui n'a d'autre but que d'éveiller la sensibilité et l'imagination, prouvent ainsi qu'aux époques où il a souverainement régné c'est l'affectus qui organise en profondeur un certain nombre de vieux ouvrages. C'est pourquoi nous devrions nous interroger sur la possibilité de construire un système critique rationnel à partir du concept d'affectus, qui peut être un instrument d'analyse opératoire. Précisons que l'affectus doit être considéré d'abord comme une zone de sensibilité qui peut être sollicitée sur tous les plans ; c'est ainsi que la miséricorde, par exemple, recouvre la bonté, la compassion, la générosité, et le pardon, alors que la justice recouvrirait la notion de bien et de mal, la justice divine et la justice sociale. Reconnaissons ensuite que c'est une valeur réversible et ambivalente, nature qu'elle doit à son origine rhétorique dans la mesure où elle est soumise à cette fameuse loi des contraires à partir d'un point de vue positif ou négatif. Dégager par exemple, dans le Guzman, une dialectique de la justice et de la miséricorde, c'est affirmer que coexistent dans la vision littéraire les deux sentiments de l'injustice et de l'égoïsme. Enfin ajoutons qu'au sein de l'acte créateur, l'affectus peut être adultéré par d'autres composantes, d'où la nécessité de distinguer des affectus dominants et des affectus secondaires. Le règne de l'affectus dans une œuvre picaresque signifie que des œuvres que nous avons trop longtemps qualifiées de réalistes obéissent non pas au besoin de décrire mais à celui d'émouvoir et qu'elles ne se soucient pas de la réalité de l'objet mais de l'impression que sa projection peut éveiller dans notre esprit. Une esthétique soumise au système des affectus est une esthétique de la théâtralité où ce n'est pas le regardé qui fait l'objet de la visée mais le regardant, où comptent les passions de l'âme du destinataire et non celle des actants et où toute la narration est prise dans l'ombre portée du narrataire. La modalité de cette présence et ses fluctuations permettent de discerner les contours de l'histoire de l'esthétique du baroque.

4 La théâtralité du Buscón est de nature bien différente. Tandis que le regard de Guzmán s'ouvre à la fois sur une intériorité et sur le spectacle du monde c'est le pseudo-spectacle offert par les gueux que dénonce le regard de Quevedo. Guzman représente le regard des marginaux ouvert sur toutes les classes sociales avec la volonté ce les cantonare dans une même unité totalisante. Dans le Buscón c'est la classe dominante qui dénonce la marginalité. Pour Mateo Aleman, la marginalité se confond avec la misère, qui est, ellemême, un facteur de désagrégation sociale; pour Quevedo, c'est la marginalité qui a occupé la place laissée vacante par la démission de la société, société qui en conséquence est, elle-même, perçue comme marginale. Mateo Aleman découvre et dévoile un spectacle, Quevedo s'efforce d'en démystifier un autre par le biais d'une mise en scène de la littérature carnavalesque dont je résumerai rapidement les phénomènes textuels: 
descriptions de banquets grotesques axées sur des éléments parodiques de la cène, mise en relief des organes d'absorption et de déglutition ainsi que des dimensions grotesques des différentes parties du corps humain dans la description qui reste le plus souvent soumise au réalisme grotesque, évocations de tripes, de ventres, de défécation, etc. cette mise à jour du système carnavalesque dans le Buscón permet de mieux comprendre les passages scatologiques dont on a rarement saisi la nature et la portée et qui, perçus dans ce contexte, retrouvent le rôle fonctionnel qui est le leur (indices des retours à la vie par exemple). Une étude structurale menée à partir de cette première approximation permet de constater qu'il y a toute une série de scènes de démystification qui sont construites sur le schéma d'une fête de carnaval qui elle, est explicite et décrite dès les premières lignes du texte. Cette fête carnavalesque est « la fiesta del rey de gallos », c'est-à-dire une fête où un jeune garçon fait office de roi et qui se termine, comme toutes les fêtes de cet ordre, par son détrônement. Sur ce schéma structural on constate que sont construites trois autres scènes aux moments essentiels de la narration. Or il est intéressant d'observer que le texte établit une homologie structurale entre cette fête carnavalesque, ces scènes de démystification d'une part et d'autre part des scènes qui décrivent la promenade à travers les rues de la ville et sous le fouet du bourreau des suppliciés condamnés par les tribunaux. Cependant lorsqu'on décide de voir de plus près comment ce système carnavalesque fonctionne dans le Buscón on s'aperçoit de la perversion que Quevedo fait subir à la perspective carnavalesque, c'est-à-dire que la faim et l'abstinence l'emportent sur la ripaille, la mort l'emporte sur la vie et la démystification (c'est peut-être le point le plus important, du point de vue qui nous occupe) - l'emporte sur le déguisement. Pris dans cet axe conceptuel, le langage dans le Buscón est perçu comme doit être considéré d'abord comme une zone de sensibilité qui peut être sollicitée sur tous les plans ; c'est ainsi que la miséricorde, par exemple, recouvre la bonté, la compassion, la générosité, et le pardon, alors que la justice recouvrirait la notion de bien et de un discours qui masque ou qui démasque et il ne semble pas excessif de prétendre que le Buscón est fait, en réalité, de deux textes qui, dans certains cas, se juxtaposent et dans d'autres cas. se recoupent. C'est ainsi que le discours mystificateur emprunte les principes de la rhétorique conceptiste mais d'une façon qui n'est pas pertinente et la non adéquation que dénonce la métaphore elle-même, est l'indice d'une usurpation. Ici. pour revenir à la perspective que nous avons suggérée à propos du Guzman tout à l'heure, c'est le regardé qui compte et on peut rappeler que le texte est véritablement scandé de la même expression (on le retrouve, sous des formes sensiblement identiques. une vingtaine de fois) : il fallait voir telle chose, « ceci était à voir etc. ».

Donc à une esthétique de la théâtralité qui serait le fait de Mateo Aleman s'opposerait une esthétique qui contrairement à ce qu'on pourrait penser à première vue lorsqu'on parle de Quevedo, démystifie l'expressionnisme, en dénonçant toutes les mises en scène qui adultèrent l'essence des êtres et des choses. Le Guzmán et le Buscón, tout en s'opposant, restent donc dans un même champ de gravitation dans la mesure où le premier témoigne d'un goût prononcé pour le spectaculaire fondé sur l'affectivité, spectaculaire que le second déconstruit en termes de réalité repoussante: mais à quelle réalité socioculturelle et socio-économique la pensée picaresque doit-elle cette structuration fondamentale de la vision du monde qu'elle propose ou qu'elle refuse et qui la fait participer marginalement ou non à l'expression baroque. À cette question je n'ai personnellement pas de réponse, je ne peux proposer que deux constatations. 
6 1-Quevedo pousse au maximum la simplification comique, déjà importante dans le Lazarillo, celle-ci devient fondamentale dans le Buscón, où l'actant est présenté sous les traits d'un bouffon tandis que Mateo Aleman la réduit au minimum dans la mesure où son narrateur est un pêcheur repenti dont le ton atteint souvent une gravité qui transgresse les lois du decorum, puisqu'il s'agit d'un gueux et d'un filou.

7 2-Quevedo, comme Mateo Aleman participent à une controverse que nous devons examiner d'une part, par rapport à l'histoire des idées et d'autre part, par rapport à révolution de la sociétéccastillanne, c'est-à-dire dans le contexte des tensions qui se produisent entre une idéologie déterminée et la réalité des faits Qui, progressivement, démolissent les bases de cette idéologie. L'axiome idéologique en vigueur, c'est que la république des hommes est organisée selon une hiérarchie immuable prévue par Dieu et donc que chacun doit admettre l'état dans lequel Dieu a voulu qu'il naisse et qu'il vive. Mais sur le plan de la réalité socio-économique, les faits ne respectent pas cette vision idéologique. La haute bourgeoisie a trahi son rôle historique en investissant ses capitaux dans l'achat de terres et de rentes Qui sont des investissements de type aristocratique. Le bourgeois castillan exprime ainsi son désir d'être noble et c'est une ambition Qu'il assouvit d'ailleurs en achetant des titres nobiliaires, d'où une inflation nobiliaire que Quevedo note avec une amère ironie. Or tandis que Mateo Aleman transcrit cette dynamique de société, Quevedo prétend au contraire démasquer les représentants d'une classe, à ses yeux ridicule, qui nourrit l'ambition de transformer une hiérarchie présentée jusqu'ici comme immuable par l'idéologie dominante. Nous résumerons leur opposition sur ce point en disant que, chez Mateo Aleman, on assiste à une réduction des différences et à une intégration des marginaux par le travail: son texte semble ouvert sur la modernité et véhicule les premiers reflets de la vision bourgeoise du monde. Quevedo au contraire accentue les différences, dénonce la marginalité comme marginale et refuse une dynamique sociale que pourtant il retranscrit. Il s'agit dans ce dernier cas d'une pensée conservatrice, et tournée vers le passé.

8 Mais le problème reste entier: peut-on considérer qu'il y a un rapport entre une esthétique essentiellement basée sur l'affectivité, esthétique qui incarne pleinement le Guzman et l'apparition de nouvelles structures mentales, qui sont elles-mêmes des produits de l'histoire.

\section{Discussion}

9 - CASTAN : Ce qui s'est passé en Espagne, ce problème du marginal et des rapports qu'une société établit avec le marginal, c'est ce qu'on voit un peu partout dans les littératures d'Europe à cette époque, en Italie, en Occitanie c'est Gaillard qui procède à une dénonciation par des thèmes carnavalesques; en Allemagne c'est Simplicissimus. Un phénomène européen.

10 - CROS : À propos de la scatologie comme symbole de vie. On en trouve beaucoup d'exemples, par exemple dans le Quichotte, Don Quichotte est censé avoir passé un certain temps chez les enchantés, c'est-à-dire dans le royaume de la mort. La première question que lui posa Sancha, c'est en gros Et là-bas, ont-ils des « toilettes ». Le monde de la mort est le monde ou il n'y a pas de défécation. La corrélation est très nette dans le Buscón: lors de l'évocation du séjour que passe Pablo chez Carra, il demande à un des jeunes gens qui est là où sont les toilettes, et le garçon lui répond qu'ici on n'en a pas 
besoin, puisqu'on ne mange pas. La corrélation est bien établie : on ne mange pas, il n'y a pas de défécation, donc on est mort. Cet épisode est d'ailleurs placé durant la période du Carême. Indiquons qu'il y a une alchimie des Cendres, à propos du Mercredi des Cendres, qui considérait que les cendres étaient l'excrément du bois.

11 - MARTINON : J'aimerais poser deux questions qui sont plutôt des interrogations à partir de votre intervention. Premièrement, j'aimerais savoir si ce qu'on appelle «le retournement du monde", aussi bien en histoire qu'en ethnologie, c'est-à-dire le renversement des rôles, l'inversion des rôles sociaux, durant une période déterminée (le roi est esclave, l'esclave est roi, l'homme est la femme et la femme est l'homme, le vieillard est l'enfant, l'enfant est nonagénaire, le fou est lucide, le raisonnable déraisonne, etc...), est-ce que ce jour des fous, ce retournement du monde était codifié, revenait cycliquement tous les ans à la même époque, était réglementé par un rituel ? Est-ce qu'il existait une réglementation du dérèglement rituel dans la société espagnole de la fin du $\mathrm{XVI}^{\mathrm{e}}$ siècle et du début $d u \mathrm{XVII}^{\mathrm{e}}$ siècle? Deuxièmement: le ventre, la nourriture, la défécation, la manière de vivre en brûlant des aliments, en les faisant circuler de l'oral à l'anal sont-ils des thèmes littéraires qu'il serait possible de mettre en corrélation avec la situation médicale, mais aussi économique, de l'Espagne du début du XVII ${ }^{\mathrm{e}}$ siècle : en un mot qu'en est-il des famines à cette époque ?

12 - CROS : Pour la première question, je n'ai pas tellement de réponse. Je ne connais pas grand-chose des scènes carnavalesques telles qu'elles se passaient dans la vie courante en Espagne. On connaît un peu plus les scènes du Languedoc, depuis les travaux de LeroyLadurie. Les scènes de révoltes se déroulent toujours dans une ambiance de carnaval, le carnaval est considéré comme une possibilité de révolte populaire et sociale.

13 - MARTINON : Il me semble que le rituel du carnaval ou du jour des fous, c'est justement une soupage de sûreté, c'est-à-dire une manière de canaliser tout ce qu'il y a de violence, en un lieu et en un temps très déterminé, avec un commencement et surtout une fin. Renverser le monde pendant quelques jours assure la stabilité normale du monde social durant toute l'année. C'est une simulation contrôlée de la révolte.

14 - CROS : Quant à votre deuxième question : c'est un problème important. Historiquement il s'agit de cette période située entre 1599 et 1604 . C'est une période de crise et de misère très grande, puisque la grande peste a sévi entre 1596 et 1602 . Elle a fait énormément de victimes: sur une population qui doit tourner autour de 5 ou 6 millions d'habitants, elle fait 500000 victimes. Elle est responsable du renversement démographique, car elle frappe les régions les plus habitées, les plus peuplées, et elle épargne les régions périphériques. C'est une période économiquement très grave pour l'Espagne. Jusqu'en 1590, il y a une montée démographique comme dans toute l'Europe. Il y a vers 1595 un essoufflement économique assez considérable, il y a des faillites nombreuses, d'autre part la peste arrive en 1596. Donc tout est réuni pour créer une problématique de la misère : il $\mathrm{y}$ a aussi à cette époque une flambée des prix, l'inflation est considérable à la fin du XVI ${ }^{\mathrm{e}}$ siècle en Castille, elle est beaucoup plus considérable que dans /es autres pays d'Europe. Pour le blé et pour le vin et l'huile, elle est de l'ordre du passage de l'indice 100 à 780 en vingt ans.

15 - DUBOIS: Un problème que je me pose, c'est celui du passage qui existe de la fête carnavalesque au spectacle. Il semble que la fête s'achève et que le spectacle commence et le personnage de carnaval est remplacé par le personnage de l'acteur. Le spectacle est tout à fait différent, dans la mesure où nous avons affaire à une répartition des rôles. Le 
carnaval est la voie réfléchie le carnaval parlerait au réfléchi. Je me déguise, je me joue ma fête. Le spectacle divise les rôles en actif et en passif : il y a des acteurs qui sont les actifs et il y a les spectateurs qui sont les passifs. Une division, un cloisonnement est établi, et d'autre part, le spectacle est toujours un lieu fermé. C'est à partir de 1600, je crois, qu'apparaît en Italie la première place payante avec l'entrée réservée. À partir de ce moment-là, il y a un cloitre. Il y a ['espace intérieur et l'espace extérieur, c'est un nouveau cloisonnement. L'opposition, au-delà de la fête et du spectacle serait la suivante : d'une part on cloisonne, il y a l'intérieur et l'extérieur, il y a l'actif et il y a le passif et d'autre part, et cela c'est le carnavalesque, rien n'est cloisonné, tout est dans tout. Il s'agit d'un immense cycle digestif qui n'est pas un cycle de dégradation, mais un cycle de production et de reproduction.

- Marguerite SOULIÉ : J'ai été très intéressée par ce que vous avez dit du passage du déguisement à la démystification, du passage du déguisement à ce qui n'est pas le déguisement : c'est ce que je trouve dans Agrippa d'Aubigné : on parle du déguisement de Satan, et toute la forme baroque aboutit à la démystification et au fait que Satan retourne à sa réalité. La même chose dans un écrit d'Agrippa d'Aubigné en prose, satirique, et qui oppose le paraître à l'être, c'est tout une œuvre de démystification. Lorsqu'on parle du baroque un peu plus tardif, alors c'est le contraire: Rousset a parlé du baroque comme déguisement et du déguisement comme création c'est le mouvement inverse. Ce que je voudrais vous demander, c'est si dans les exemples que vous rencontrez, les marginaux sont considérés comme les intermédiaires entre le monde de la réalité et un monde du rêve, de la magie ou du fantastique.

17 - CROS : Je ne connais pas d'exemple de médiation de ce type. Le gueux revendique tout au contraire un rôle de narrateur: guide qui dévoile la réalité, dans le Guzman, il s'intitule le « guetteur de la vie humaine » (atalaya de la vida humana), qui, du haut de sa tour de vigilance décrit le théâtre qui se déroule à ses pieds.

18 - COURTÈS : L'exposé de Cros m'est apparu comme une leçon de méthode. Parce qu'il est venu, de son propre aveu, avec un passeport d'étranger aux rencontres sur le baroque, il s'est interdit d'aborder des problèmes de définition. De ce fait son projet n'a pas eu à souffrir du cadre dans lequel nous aurions pu l'attendre.

De là encore la construction particulière de celui-ci. Les concepts n'y font pas office de garde-fous mais de passages. Ils sont suivis comme des chemins et non pas comme des frontières. Fabliaux, vie matérielle, situation économique dessinent le courant populaire jusqu'au lieu de sa rencontre avec la rhétorique. Nous avons été mis en présence d'une carte qui était celle d'une confluence ; l'illusion, la dénonciation, le dialogue ou la dualité ont pu jouer ainsi leur rôle fondateur, celui qui donne naissance aux significations: l'affrontement de Quevedo avec Mateo Aleman, celui-là écrivant à l'heure où celui-ci est publié, en apporte une illustration réellement privilégiée. Je voudrais préciser deux points qui se rapportent à la pratique de cette méthode.

21 On a parlé de scatologie. L'objet qu'elle désigne, pris au sens naturel, n'est qu'un phénomène de la vie. Le tout est d'identifier les mythologies successives ajoutées à ce degré zéro. L'exemple rabelaisien est tellement différent de ce qui vient d'être évoqué que la comparaison n'a plus rien d'instructif : en lui la même jovialité transfigure le corporel, qu'il soit alimentaire, génital ; urinaire, athlétique. La vérole pourrait y être action d'éclat, fanfaronnade, absolument pas châtiment. La farce y est animée d'exaltation épique: tout le contraire de la parodie picaresque, fascinée par l'horreur (ou 
le désir) du blasphème. La prouesse et le bûcher : décrochage historique ? Encore le Moyen Age, déjà la Contre-Réforme. Pourquoi pas géographique ? La grossièreté de ces explications fantastiques est refusée par la richesse des variantes propres au domaine espagnol. Les enchantés du Quichotte sont réduits à l'état de chant: or il n'y a pas d'allégement (pas d'exonération) pour des êtres sans poids ; Cervantès suit la loi logique du discours. Quevedo suit une loi tout à fait différente, celle du regard scandalisé, force lui est de préférer qu'il n'y ait rien à voir : l'abstinence vaut mieux que la ripaille parce qu'elle nous dispense d'un spectacle écœurant.

Quevedo est le prophète qui dit comme elles sont les choses qui ne devraient pas être: porte-parole de l'Éternel, indigné, ulcéré des horreurs de son temps. Il est l'accusateur, dont le réquisitoire comporte un éloquent chapitre sur l'argent. Comme toujours, le sens de celui-ci est porté par sa différence. Lié aux biens de consommation, il représente pour l'usager privilège, privation exclusion, hiérarchie. Au contraire pour Quevedo, lié à l'éclat du visible l'or ne peut que symboliser le déguisement destructeur, la profanation par laquelle toute chose perd son essence. L'or que l'on va chercher, qui n'est pas un produit, mais un corps étonnamment stable, abandonne et trahit l'Espagne après l'avoir désagrégée: la crise qu'il provoque en réclame toujours plus, mais il ne fait que passer ; les banquiers de Hollande l'appellent dans leurs coffres, où il emporte la substance du pays qu'il a épuisé. L'or ignore le distinctif : les nations, les personnes ; les fonctions aussi bien. D'où le pouvoir de simulation qu'il confère à la bourgeoisie prompte à quitter son rôle moteur et directeur pour la figure, ou le contresens des conduites conservatrices, des titres de stabilité (illusoires, bien entendu) antérieurement réservés à l'aristocratie tutélaire. L'or est le signe universel dont l'abstraction ensevelit le particulier des raisons d'être, et par là décompose leur association qui fut une cité cohérente. Visionnaire ou témoin du naufrage des formes, Quevedo invente ou cautionne la réaction désespérée d'une droite anti-capitaliste, dont la sincérité peut malheureusement elle aussi faire l'objet d'une simulation.

23 C'est pourquoi il lui faut une autre dimension que celle de l'actualité. Sans doute Quevedo cache-t-il son regard au bénéfice du regardé: mais ce changement fait apparaitre la manière de voir de Mateo Aleman. Si la justice est un affect, au lieu d'être comme chez Platon la vertu de communiquer, la liaison commune aux vertus différentes, c'est que les différences sont devenues apparence, que le destin des formes est de communier dans l'indistinction de leur principe (ou fondement ou origine) : les humbles et les grands seront récupérés, les fautifs réhabilités. La réponse de Quevedo est que si les différences sont démystifiées, la vérité (qui est différence) est anéantie du même coup; l'acte démystificateur doit être alors démystifié. Si la société n'est plus ce qu'elle doit être, faute d'une répartition harmonieuse des rôles, le peuple cesse d'être peuple comme l'aristocratie d'être aristocratie. Sa protestation contre l'informe est celle (peut-être anachronique) d'une théologie thomiste, médiatisante, contre une autre théologie, augustinienne, nominaliste. Difficile, mais nécessaire, le repentir est un chemin, comme la distribution sociale des missions (travailler, combattre, prier) organise le salut selon une méthode : spirituel ou social, l'homme est un pèlerin. L'aberration du gueux consiste à se donner, sans aucune distance, toutes les formes à la fois. C'est ainsi que je comprends le titre de Protée, qu'Edmond Cros naguère a choisi pour ses recherches sur le marginal (en qui nous reconnaissons un personnage baroque): contre quoi Quevedo proteste que le gueux, qui n'est rien vraiment, ne peut pas non plus être un vrai gueux. Le Guzman et le 
Buscón donnent donc la parole à deux archétypes contraires de la théologie chrétienne, le plus baroque étant à mes yeux le premier.

$24-$ CROS : Je suis très sensible à ce que tu as dit à propos du thomisme, mais je n'en vois pas trace chez Quevedo, par contre qu'il y ait eu une influence augustinienne dans le Guzman est un fait attesté.

- COURTÈS : La signification sociale du thomisme a cependant été reconnue de très bonne heure. Si de nos jours on juge apocryphe l'un des textes où elle est stipulée, ce n'était pas le cas au temps de Quevedo. Son existence même prouve la facilité d'une traduction politique de la Somme théologique, sur la base d'une opposition aux théologies de l'immédiat. Pour celles-ci la notion d'un Dieu conservateur fait de notre simple présence une affirmation de la sienne : il est l'âtre de tout ce qui est. Chez Saint Thomas les preuves s'éloignent : il y a seulement des voies d'approche, par quoi nous prenons conscience que nous ne sommes jamais au but. Or la route nous fait marcher: tout ce qui parle d'itinéraire (médiation ou méthode) contient donc, au moins comme menaces, notre aliénation et notre séparation.

Pour nommer le désaveu de cette formalité pénible il ne va pas de soi qu'on pense à la réforme. D'une part en effet le mot peut induire en erreur : s'il n'est pas entendu comme restauration, revendication, renaissance du christianisme originel, il peut suggérer par mégarde le rétablissement d'une rigueur. D'autre part il y a, comme on dit, tout un monde entre la liberté dans la déréliction et la liberté du chrétien. Or justement il y a un monde, c'est-à-dire une cosmologie où la forme des phénomènes, l'apparence où ils persévèrent, a moins de prix que cette persistance comme manifestation de l'alliance divine. Il fallait bel et bien une foi augustine, celle de Luther, pour engager la pétition théologique en faveur de l'immédiat, depuis laquelle le désespoir, la tendresse, la miséricorde confessent leur intime parenté.

S'il était permis de revenir sur le mot code prononcé hier, je dirais qu'il n'est pas essentiel au baroque, mais s'il est vrai que l'absence de Dieu soit le châtiment de ce monde, le seul ordre qui soit alors à sa mesure est celui de ses correspondances, éventuellement paradoxales: à la limite du pour au contre, comme aux rites du carnaval. Ainsi la thématique monétaire est une voie, c'est-à-dire une institution, dans laquelle n'importe quoi peut induire n'importe quoi, sans médiation intrinsèque. Cette codification foncièrement quelconque, cette caricature de la nécessité, secrètement identique en son absurdité à la liberté de Don Juan, constitue donc évidemment un aspect provocant de la modernité : la réaction de Quevedo fait preuve, sur ce point, d'une parfaite cohérence.

- LAFAY : M. Cros fait l'économie du baroque d'une part et d'autre part il insiste sur la réalité socio-culturelle et socio-économique, c'est-à-dire qu'il établit un lien d'explication et de relation entre la réalité socio-culturelle, socio-économique et la réalité littéraire. Je me demande si ce n'est pas précisément parce qu'il a cette explication socio-économique qu'il fait l'économie de la catégorie du baroque. On peut en effet la concevoir de différentes façons. Ou bien cette catégorie du baroque est une catégorie esthétique et mentale qui servirait de médiation entre les réalités socio-économiques, socio-politiques et la réalité littéraire. En ce cas, on peut faire plusieurs hypothèses: si l'analyse de la réalité socio-culturelle est plus large, plus profonde que celle de la catégorie esthétique et mentale du baroque, la voie socio-critique que vous suivez rendrait inutile ce passage par le baroque; si c'est la catégorie du baroque qui est plus large, il faudrait y insérer la composante socio-économique sur laquelle vous insistez; à moins que la réalité culturelle du baroque s'enracine dans la réalité socio-économique pour un enrichissement mutuel. 
Ou bien on peut exagérer la difficulté et dire qu'il n'y a pas compatibilité entre une analyse et l'autre, entre d'une part l'étude socio-critique qui est votre perspective et qui cherche un enracinement historique au sens large et d'autre part le baroque, si le baroque est conçu dans la perspective dorsienne, forme universelle apparaissant comme un leurre idéaliste pour un tenant d'une analyse socio-historique.

L'analyse du baroque se situe selon moi au niveau des structures mentales qui sont enracinées dans la réalité socio-économique, projetées par elle. Même ce qui, de la réalité littéraire, est analysé en terme d'héritage reçoit sa vraie signification de sa relation à l'entour socio-culturel d'une époque donnée. Ce qui est important, c'est la façon dont cet héritage est utilisé et finalement transformé ; peu importe qu'on imite Horace ou qui l'on voudra, ce qui compte, c'est l'usage littéraire qu'on en fait et cet usage s'analyse à partir des réalités mentales enracinées dans la réalité culturelle large, elle-même liée à la réalité historique. Selon qu'on met l'accent sur la dimension d'héritage ou sur la dimension d'insertion dans une réalité socio-culturelle et historique, on considère que la littérature engendre la littérature ou au contraire que c'est une réalité autre que littéraire qui donne à chaque période à la littérature un visage nouveau. Dans ce croisement du littéraire hérité et du présent historique, donne-t-on plus d'importance à l'engendrement de la littérature par la littérature ou aux transformations apportées par l'insertion dans la nouvelle réalité socio-historique caractéristique d'une certaine période?

On retrouve alors le problème difficile qu'on évoque depuis le début, celui de la périodisation. Je ne suis pas sûr qu'il soit toujours bien posé, parce que par périodisation on entend trop souvent une tranche chronologique qui se définirait par un fonctionnement relevant d'un seul système mental. Mais précisément à l'intérieur d'une tranche chronologique ne fonctionne pas forcément un système mental unique (l'unité du xvII ${ }^{e}$ siècle classique n'existe pas au début du siècle); il peut y en avoir plusieurs. Et, enfin, tout cela ne s'arrête pas brusquement ; les évolutions qui engendrent les mutations sont progressives; une structure socio-culturelle peut être en voie de déstructuration et être encore très pesante, et une structure culturelle peut être en structuration et n'être pas encore quantitativement dominante. Cela devrait conduire à faire des coupes en biais, qui régleraient les problèmes de décrochages dont on parlait tout à l'heure, encore qu'il s'y ajoute un problème non plus seulement temporel mais aussi géographique.

31 -MARTINON: Il me semble qu'on peut tirer quelques conclusions en formes d'interrogation de ce qui vient d'être dit dans les interventions précédentes.

J'aimerais surtout m'interroger sur cette idée de Laboratoire imaginaire du baroque chère à Félix Castan. Et mettre en rapport cette volonté et cette demande de la création d'un laboratoire imaginaire, avec ce qui a été dit à propos de l'apport des diverses disciplines que nous représentons, à cette œuvre de compréhension et d'analyse du baroque : toute discipline « sans exclusive » est partie prenante dans une telle demande et dans une telle élaboration. Il s'agit donc d'un laboratoire de types pluridisciplinaires acceptant toutes sortes de méthodes utilisées afin d'appréhender l'objet «baroque », d'un laboratoire imaginaire puisque ses membres sont insérés dans des institutions diverses, Universités, CNRS, EPHE, etc., qu'il ne s'agit donc pas de la création d'une nouvelle institution de recherche mais d'un lieu de rencontre servant à présenter et à confronter les divers états de la recherche sur le baroque. Mais il ne s'agit pas seulement du problème de la diversité des institutions et des recherches, c'est-à-dire d'un problème d'histoire de la recherche, mais aussi d'un problème épistémologique. En effet, le concept de baroque provient d'un phénomène culturel, littéraire, artistique, architectural, musical, de vie quotidienne, 
théologique et se développant historiquement, se situant géographiquement. Pour le comprendre, on peut utiliser l'analogie astronomique de la lune et du soleil. Je veux dire par là que nous avons affaire à de l'empirique catégorisé par une définition sociale comme la lune et le soleil sont perçus empiriquement par le regard mais peuvent être aussi objet de sciences: la sélénographie, l'héliographie. Cette comparaison n'est pas nouvelle. Passeron en parle longtemps dans de métier de sociologue ». Mais ma question est alors la suivante. Le baroque, empiriquement, peut-il être constitué comme objet de science. Je ne le crois pas: c'est un fait socio-culturel, un fait total justiciable de plusieurs approches qui, elles, peuvent être scientifiques. Il n'y a pas de-science du baroque mais il y a des discours scientifiques qui peuvent s'interroger sur le baroque. Histoire, sociologie, psychanalyse, économie, esthétique, science de la littérature, linguistique, philosophie, etc. Vous voyez le rapprochement avec l'héliographie ou la sélénographie qui sont des sciences utilisant, pour la compréhension de leur objet, d'autres sciences dites théoriques, physique, chimie, etc. Le baroque est dans le même cas, si je puis dire que la lune et le soleil : pour être compris on doit faire appel à des sciences humaines à prétention théorique, sociologie, philosophie, psychanalyse, économie, etc. Or tous ces discours ne définissent pas de la même manière le phénomène baroque ni historiquement, ni géographiquement, ni structuralement. N'assistons-nous pas alors à une dissolution de la notion même de baroque, la sommation des études sur le baroque ne formant pas structure. En effet qu'y a-t-il de commun entre le discours du psychanalyste s'intéressant à la création littéraire et l'économiste parlant du flux des métaux précieux. Lorsqu'ils parlent d'or, ce ne sont pas des mêmes pièces, du même métal qu'il s'agit. Dissémination des discours et dissolution de l'objet baroque en une constellation de petits objets Spécifiques traitables par les diverses sciences humaines. Voici une voie, désespérante peut-être, de la recherche. L'autre voie c'est justement celle de la confrontation et non pas de la sommation à tout prix, des divers apports des travaux scientifiques. Cette confrontation devant être le lieu de travail qui, sans construire a priori son objet: le baroque, essaye de découvrir - au niveau du bricolage et non pas de la vaste synthèse comment toutes ces approches peuvent faire système pour comprendre un fait social total qui serait le baroque.

Donc un laboratoire qui ne serait ni un musée collectionnant les interprétations et les travaux sur le baroque. ni un univers carcéral de la définition a priori (hors de la définition qu'on donne du baroque point de salut) ni non plus un capharnaüm dans lequel tout serait dans tout. Nous sommes plutôt dans une phase de circulation de l'information sur le baroque et non pas dans une phase d'élaboration d'une théorie unique pouvant nous faire comprendre ce qu'est le baroque. L'ironie du baroque étant peut-être de nous confronter à la démesure de notre tâche de compréhension de ce phénomène culturel. Tout ceci pour dire que rai très peur qu'une théorisation hâtive du baroque comme catégorie esthétique et mentale tel que peut le penser Eugenio d'Ors n'est qu'une condensation imaginaire d'un champ empirique non déterminé. Au-delà des situations historiques géographiques sociales, il y aurait un discours "à propos de ", explorant des catégories sans lesquelles des objets culturels hétéroclites pourraient se ranger. Les sculptures des temples de Kharjuraho aux Indes, ou de Borobudur à Java, deviendraient baroques comme l'église Saint-Yves de Borromini. Mais alors, pourquoi pas l'art des fous, le facteur Cheval, etc. Cette manière de penser me semble totalement fascinante car elle tente d'expliquer ce qu'est le transculturel, de rejoindre par là des préoccupations de type universel, mais très dangereuse car illimitée dans son contenu. Et si l'on prend un concept ethnographique, on pourrait dire que c'est de la magie sympathique au niveau 
conceptuel. Cette dissémination dans l'hétéroclite des objets empiriques étudiables sous le mot baroque me semble très dangereux dans la mesure où nous n'avons pas affaire seulement à une dissémination mais une dissolution totale du champ.

34 - CROS : Il me semble dangereux de vouloir s'en tenir à une périodisation très précise car lorsqu'on parle de Rabelais, lorsque celui-ci exprime des structures mentales de la société, ce sont celles de son milieu, etc. Il est bien évident qu’à la même époque nous ne trouverons pas la même société en Espagne ou en Italie et, il faudrait réussir à définir une période modèle et d'en voir la composante, les structures mentales, etc., et, à partir de là, voir si ['on retrouve dans une société voisine, peut-être à dix ans de distance, les mêmes composantes.

35 - DUBOIS : Je voudrais savoir ce que c'est qu'une structure mentale. "Avant donc que d'écrire apprenez à penser » dit Boileau. Or quand récris, je pense, c'est la même chose, on écrit en pensant et on pense en écrivant. Si on me dit d'abord on pense et ensuite on écrit, je vois ce que cela veut dire. C'est une pensée qui s'exprime en mots. Une structure psychique je vois aussi, mais une structure mentale je ne vois pas ce que cela veut dire et ce que cela ajoute au terme penser. Est-ce que cela serait antérieur et extérieur à la structure verbale? Le deuxième problème concerne les rapports du baroque et de l'économie. Il y a une économie que l'on peut qualifier de baroque. Un type d'économie classique, on peut en donner une définition, c'est la mise en valeur ordonnée d'un capital hérité, c'est la parabole des talents. Le père donne des talents à ses fils et dit : gardez-les. Deux investissent le capital reçu, le troisième enterre le talent. Il me semble que ces deux attitudes peuvent symboliser l'économie de type classique. On investit le capital dans le circuit économique ou alors, et c'est une autre forme de l'économie classique, c'est le conformisme, et l'académisme : on le garde et on le conserve.

Il y a une autre parabole qui pourrait avoir une interprétation socio-économique et je me demande si celle-ci ne définit pas une économie qu'on pourrait appeler baroque. Percevoir un capital et le dépenser : c'est ['enfant prodigue. Il fait valoir son capital, mais pas en l'investissant, en le transformant en apparence. Est-ce que l'économie baroque, ce ne serait pas ça. Une manière de recevoir un capital et puis de le dépenser, de le faire valoir, ce qui mettrait en jeu le rôle de la classe aristocratique, qui est une sorte d'agent publicitaire : il y a des produits et ces produits sont consommés par des aristocrates qui font valoir ces produits. Si on transpose, dans le domaine littéraire, ce faire valoir par la dépense, ne serait-ce pas une manière d'utiliser le mot pour en faire de beaux feux d'artifices, des redondances rhétoriques, des accumulations verbales, l'usage de l'hyperbole, tout cet usage de la langue qui rejoint l'usage économique qu'ils ont fait des produits matériels.

- MARTINON : C'est très séduisant, mais comment en apporter la preuve? 


\section{AUTEUR}

\section{EDMOND CROS}

Professeur d'Espagnol Centre d'Études et de Recherches Sociocritiques Université de Montpellier 